\title{
Multilinguales
}

12 | 2020

Varia 2019

\section{Résurgence mythique et réflexion ontologique chez Malika Mokeddem}

Mythical resurgence and ontological reflection at Malika Mokeddem

\section{Sabrina Tounes Yamouni et Souhila Ourtirane Ramdane}

\section{(2) OpenEdition}

Journals

Édition électronique

URL : https://journals.openedition.org/multilinguales/4206

DOI : $10.4000 /$ multilinguales.4206

ISSN : 2335-1853

Éditeur

Université Abderrahmane Mira - Bejaia

Référence électronique

Sabrina Tounes Yamouni et Souhila Ourtirane Ramdane, «Résurgence mythique et réflexion ontologique chez Malika Mokeddem », Multilinguales [En ligne], 12 | 2020, mis en ligne le 14 février 2020, consulté le 30 juin 2021. URL : http://journals.openedition.org/multilinguales/4206 ; DOI : https://doi.org/10.4000/multilinguales.4206

Ce document a été généré automatiquement le 30 juin 2021.

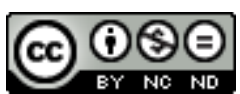

Multilinguales est mise à disposition selon les termes de la Licence Creative Commons Attribution Pas d'Utilisation Commerciale - Pas de Modification 4.0 International 


\title{
Résurgence mythique et réflexion ontologique chez Malika Mokeddem
}

\author{
Mythical resurgence and ontological reflection at Malika Mokeddem
}

\author{
Sabrina Tounes Yamouni et Souhila Ourtirane Ramdane
}

1 Afin d'interpelerson lecteur et de pourvoir une lisibilité particulière, chaque roman mobilise des stratégies scripturaires singulières assurant la fluidité du récit et son intelligibilité. Selon sa matière idéologique, son rapport au temps et à l'espace, le texte littéraire s'offre toujours le plaisir mais surtout le moyen d'engendrer ses propres procédés de signifiance, de tisser son épaisseur et de marquer sa propre identité. Qu'il ait recours à certains motifs littéraires connus ou à quelques mythes anciens ou nouveaux, le récit fait jaillird'emblée un parcours ontologique qui se fait et se défait au fil de son écriture et /ou de sa lecture.Ce faisant,l'écriture romanesque peut se saisir d'un croisement de pratiques narratologiques et de jeux rhétoriquespour créer des territoires divergents, réveiller des imaginaires ou développer des dessins inhérents à l'expérience et à la culture de l'auteur d'une œuvre.

De façon similaire, la reprise d'un mythe au sein d'un récit implique le plus souvent une double expérience. Au niveau le plus simple, la critique littéraire affirme que le recours au mythe est un moyen d'emprunt exogène et endogène au texte littéraire. Pour cette double approche de l'écriture littéraire du mythe, le texte demeure l'itinéraire inscrivant poétiquement un alliage savoureux entre projet individuel et des bribes de mémoires hétérogènes. Le récit littéraire, conjugué au mythe, engendre alors un espace de voyage, d'hybridité, de piochement des consciences fugitives, de recueillement et de reconstruction selon la critique littéraire Marie-Chatherine Huet-Brichard :

«[...]le mythe est à la fois une référence hors-texte, qui appartient à un autre champ du domaine culturel [...]et un élément constitutif du texte. Parce qu'il est dehors et dedans, étranger et semblable, ses liens avec la littérature relèvent de l'étrangeté » (04)

2 Au reste, par sa force de générer des fusions et des digressions, la convocation d'un mythe dans une œuvre littéraire produitun syncrétisme oùdes cultures et des ramifications diverses de modes de penséese disséminent pour développer une œuvre 
arpentant plusieurs passions et inhibant sans ambages la pensée paresseusequi célèbre l'enfermement. L'écriture et /ou réécritured'un mythe littéraire s'allierait, dans ce sens, à la problématique de l'intertextualité ${ }^{1}$ développée par la critique Julia Kristeva selon laquelle l'œuvre littéraire-riche en emprunts - fragiliseirrémédiablement le particularisme pour prôner une œuvre habitant différemment des univers disparates.

Dès lors, il demeure inestimable de repositionner les œuvres littéraires au cœur des approches critiques renouvelant le regard sur les enjeux textuels et/ou culturelsvéhiculés par le texte littéraireet d'une nouvelle identité qui se surplombe pour être étrangère à elle-même $e^{2}$ faisant ainsi d'un livre un territoire où se laisse couler une convergence entre énergie individuelle et bifurcations symboliques universelles.Dans cette perspective, nous relevons dans La Désirante (2011) de Malika Mokeddemdes errances plurielles pour témoigner d'un brassageculturel et/ou d'une identité dont l'essence serait la quête d'un humanisme avéré. Nous pensons que l'origine de ces errances est le mythe d'Ulysse dont nous tenterons d'identifier l'investissement scripturaire à partir d'une mise en évidence de ses mythèmes. Ces derniers accompagneraient, bel et bien, la subjectivité de l'auteure de La Désirantepour poétiserdans l'espace du tissu intertextuelun nouveau scénario mythique et une aventure singulière qui se surpasse.

3 En examinant de plus près le texte de La Désirante de Malika Mokeddem, on parvient à avancer que l'errance, côtoyant jalousement le désir d'évasion,inscrite en amont et en aval du roman,par delà son caractère mystique gagnerait dans pareil cas à être un support probant d'un projet autobiographique fragmentaire faisant dévoiler une écriturerévélanttumultueusement une mémoire identitaire qui s'exhume de la contingence.

Dans cet article, notre propos est de montrer que l'errancetextuelle en rapport avecle traitement thématique dumythe d'Ulysse fait du roman La Désirante un espace discursifmettant en exergue l'alliance poétique entre deux espaces géographiques mais surtout identitaires qui sont la mer et le désert. D'où ces questions : par quelles stratégies d'écriture se manifeste le lien entre quête identitaire et langage mythique? Quels liens existent-ils entre l'intégration textuelle du mythe d'Ulysse, l'identité du personnage et la poétique de l'errance ? La reprise du mythe d'Ulysse s'accorde-t-elle avec le texte homérique?

Nous tenterons de répondre à cette problématique, en émettant l'hypothèse suivant laquelle: les multiples dimensions de l'errance accompagnent souvent l'errance scripturaire en vue d'une transcendance identitaire et/ou poétique.

4 A l'image des confusions identitaires dont souffrent la plupart des personnages des romans de Malika Mokeddem,l'errance notamment dans le désert cautionne le projet autobiographique confectionné au sein du récit de La Désirante incarnant par conséquent la tradition ancestrale de l'auteure .En dernier ressort,la mise ne fiction de l'errance s'emploierait ex abrupto à traduire aussi bien une césure identitaire du personnage principal juxtaposée à la perte et à la bâtardise ainsi qu'un phénomène d'interculturalité du fait de la double appartenance de l'auteure du roman et ce, sans oublier l'errance textuelle qui afficherait pour sa part cette volonté de produire de nouvelles signifiances. 


\section{La désirante entre configuration spatiale et langage identitaire}

5 Essentiellement ambivalent, l'espace de La Désirantede Malika Mokeddem s'offre comme un espace d'ancrage et d'errance et laisse saisir une poétique où déferle la métaphore de la mer et celle du désert. Deux espaces, d'emblée contradictoires, accompagnant de façon indéfectible la quête identitaire du personnage principal qui raconte longuement les histoires de ses errances.

En effet, c'est dans la complexité du portrait de Shamsa, narratrice homodiégétique qui tente de trouver un équilibre existentiel dans l'espace religieux où elle a été élevée par des sœurs blanches, après son abandon, que peut se comprendre la relation à l'espace et aux êtres. En reliant l'histoire personnelledu personnage à l'espace, la narration dévoile la profondeur des défis proposant de nouveaux recommencements. De ce point de vue, l'espace de La Désirante, foncièrementmarqué par son ouverture, relève d'une extension significative du personnage principal qui ne comprend guère les motifs de son étrangeté. En outre, l'errance entre un milieu thalassique (la mer) et un milieu minéral(le désert)permet de transcrire dans l'espace du récit la dislocation psychologique du sujet de la fiction (Shamsa), de teindre une rhétorique de retranchement et d'offrir à l'héroïne de La Désirante- qui ne s'identifie dans l'espace que de façon imaginaire -l'opportunité de déplacer son regard par rapport à son passé et d'exhumer son identité méconnue.

6 Portant au plus profond d'elle son » inquiétante étrangeté » car exclue dès son enfance de son désert, Shamsa sculpte, suivant ses désirs, les espaces où elle évolue et s'élance -à son corps défendant -dans le rétablissement de sa mémoire traumatique. Du même coup, la fiction se construit autour des errances spatiales mais aussi virtuelles de Shamsa se dédoublant ainsi à sa quête identitaire.

L'espace géographique apparaît dès lors comme une fresquesculptée à partir de la mobilité de Shamsa. Dans ce contexte, le texte de La Désirante, en inscrivant l'intertexte homérique, offre au lecteur des incursions dans différents registres temporels et reconstruit ses propres espaces mythiques à l'instar de l'espace de l'écriture. A ce titre, tous les emboîtements sont motivés par les errances de Shamsa dont la destinée viatique rappelle à souhait celle d'Ulysse. Lorsque les anachronies s'opèrent, dans le récit de $L a$ Désirante, le roman de Malika Mokeddem s'offre à l'art de la réécriture des épopées anciennes. En fait, la métaphore de l'errance émerge et tient toute sa révérence dès les premières pages du romanainsi que dans l'épigraphe empruntée à Saint John Perse dont voici le contenu :

«C'étaient de très grands vents sur toutes faces de ce monde, De très grands vents en liesse par le monde, qui n'avaient d'aires ni de gîte, Qui n'avaient garde ni mesure, et nous laissent, hommes de paille, En l'an de paille sur leur erre... » ( Perse dans Mokeddem, 2011.)

7 Transportée dès sa naissance dans le désert algérien par un camion lors d'une tempête de sable, Shamsa devient spontanément un être errant. C'est ce que l'on peut examiner dans les propos de la narratrice intégrant l'histoire de Léo, son compagnon disparu, à son histoire personnelle :

Pendant que tu me racontaiscela, une voix claire entremêlait un autre récit du désert au tien, celui de mon histoire :tapi dans l'obscurité, quelqu'un avait épié le chargement d'un camion[...]Un papier glissé entre les plis de mes langes[...] 
disait : » elle est née dans la nuit.Sauvez-là s'il vous plaît. » [...] ainsi transporté par

un jour de grand vent à travers le désert. (Mokeddem, 2011 : 56-57)

Par ailleurs, Malika Mokeddem en convoquant régulièrement la pensée de Saint-John Perse dans ses romans - notamment la thématique du vent - laisse se profiler par bien des aspects une affinité avec la symbolique de l'errance propulsée par le déchainement $d u$ vent $^{3}$ et renforce, par extension, tout l'imaginaire relatif au désert.

8 Cela dit, le vent demeure un motif signifiant travaillé poétiquement dans le roman $L a$ Désirante.De la tentative de prénommer Shamsa "Vent de sable » au nom du bateau de Léo "Vent de sable » - même appellation du bateauque l'on retrouve dans le roman Mes Hommes de Malika Mokeddem -le vent met au diapason toute une constellation de souvenirs, devient le cadre intime du récit et rattache, de façon métonymique, tous les autres éléments textuels au désert. De telles affirmations se laissent entendre dans les propos des personnages du roman La Désirante :

« Il faut plutôt la surnommer Vent de sable. Plaisanta l'une d'elles. " (Mokeddem, $2011: 58)$

Plus loin dans le roman, le vent est encore convoqué car il ramène inéluctablement à une rêverie sur le monde de l'enfance :

" Il s'appelleVent de sable.Je ne pouvais pas le baptiser autrement. » (Mokeddem,

2011 : 53-54), réplique Shamsa.

9 Ainsi, la rhétorique du désert implique des révélations identitaires sur l'auteure de notre roman ainsi que sur son personnage. Plus qu'une simple description spatiale, l'évocation du désert dévoile une écriture de l'errance prenant le décor désertique comme espace d'identification des personnages du roman La Désirante.Désormais, la présence du désert dans le récit de Shamsa place le système sémiotique de la narration à la jonction de l'enracinement et de la liberté. Pourainsi dire, toute la poétique de La Désirante met en toile de fond le nomadisme prenant tout son sens dans l'enfance de Shamsa.

En effet,l'espace tragique où évolue cette héroïne la projette précocement dans le mouvement :

" Je suis née d'une tombe de sable. La mer est mon désert .Toi mon port d'attache.»

( Mokeddem, 2011 : 74)

D'autre part,la mer devient le lieu initiatique dans lequel se réalise la quête de Shamsa dont l'errance est accolée à celle d'Ulysse par le recours à la référence ${ }^{4}$ explicite de la narratrice au personnage mythologique :

«Mes traversées sont celles d'un Ulysse sans Ithaque. Ulysse, quand les cris de milliers de naufragés remplacent le chant des sirènes. Tu es nulle part. Vais-aller de Charybde en Scylla?» (Mokeddem, $2011: 155$ )

10 En revanche, la réécriture du mythe d'Ulysse dans La Désirante demeure subversive en rapport autexte homérique car la problématique du non retour est incessamment évoquée dans le roman.Au-delà de l'errance spatiale, toutes les mises en discours laissent transparaître une démarche singulière de l'altérité. En fait, Shamsa, en manque d'affiliation parentale précise, se cherche tout au long du roman, esquisse son autoportrait à travers son discours sur les autres et entame des pérégrinations lui permettant d'édifier un chemin vers le bonheur et de porter au pinacle une identité interculturelle ${ }^{5}$, une entreprise ne pouvant se réaliser que dans l'espace du texte.Suite à la disparition de Léo, une introspection s'impose dans la mémoire de Shamsa :

«Ce voyage à ta recherche est aussi une façon de me réconcilier avec Vent de sable, de ne pas le dépérir, de l'impliquer dans ma première traversée seule. De reprendre son vent dans ce but. » (Mokeddem, $2011: 33$ ) 
Dans cette optique, la narration explore l'effet mythique d'une dialectique spatiale assez récurrente dans les romans de Malika Mokeddem. Cela dit, le parallèle entre la mer et le désert, la ville et le voilier s'accomplit pour arriver non seulement à percer le mystère de la quête identitaire mais aussi à faire revivre le désir d'exister et ce, en s'adonnant aux grâces de la nature :

«La mer est mon désert » (Mokeddem, 2011 : 75), confie Shamsa à Léo.

La mer serait conjointement aux yeux de Shamsa :

«le côté face de l'amour " (Mokeddem, $2011: 213$ )

11 Conséquemment, l'espace géographique amplifie inéluctablement les éléments de la diégèse, offre aucadre durécit des accents mythologiques et engendre un enchevêtrement textuel atypique. Enfin, l'espace constitue le procédé métonymique de la révélation identitaire, crée une affectivité incandescente chez le personnage principal et permet unrattachement surprenant entre sensibilité poétique et sensibilité intime. D'autre part, l'errance à travers des espaces multiples fait miroiter le nomadisme intérieur dont se réclame Shamsa :

«De déplacement en déplacement, je demeure nomade dans l'âme et garde en moi ce quelque chose qui fait que les grands espaces me dévastent. » (Mokeddem, 2011 : 93)

« Ici seuls les palmiers ont des racines. » (Mokeddem, 2011 : 95)

Par ailleurs, les différentes traversées de Shamsadévoilent plusieurs digressions sur son traumatisme primaire qui est, entre autres, celui de l'abandon. L'écriture de l'espace transpose ainsi des questions identitaires et permet d'imprimer les interstices d'une histoire manquée. Sans aucun doute, la poétique spatiale procède de ce pouvoir à conférer au texte de La Désirante le caractère d'un récit de mémoire édifiésur la nécessité de retourner aux sources. Une nostalgie de retour qui se dégage sur le plan del'écriture par des métaphores ingénieuses à l'image de ce navire baptisé «Vent de Sable » qui rappelle inéluctablement que Shamsa n'est que la fille du désert.Au-delà du récit de la mémoire, l'espace discursif du roman $L a$ Désirante cultive aussi l'errance ente les cultures et l'errance entre les textes du fait que Shamsa ponctue non seulement son journal intime par un discours sur sa société d'origine tout en ne manquant d'évoquer les problèmes inhérents à la région du Sahel mais elle multiplie aussi les références à d'autres textes littéraires issus d'autres aires culturelles à l'exemple de la référence au texte filmique d'Alfred Hitchcock, en l'occurrence : «Les oiseaux. »

D'autre part, la mer devient l'univers symbolique qui permet à Shamsa une parfaite sécurisation de son univers affectif et lui donne une capacité à se régénérer et à faire le vide :

J'avais gardé l'habitude de courir vers la Méditerranée.Pour faire le vide.Le miroir des eaux chassait mes hantises[...]Tant d'années à me tenir juste au bord de ressac ,à part,toute entière dans le souci d'humer la brise du large,d'éprouver la solitude dans le murmure de la mer comme dans ses colères. (Mokeddem, $2011: 62$ )

13 Nonobstant le contact rassurant avec l'eau, le passé saumâtre de Shamsare fait surface car en franchissant les eaux de la mer l'héroïne se trouve une nouvelle fois confrontée à des vérités amères à savoir celles de son abandon par sa mère. De ce fait, le récit donne libre cours à l'expression originale du «je » pour concrétiser des métaphores de la présence et de l'absence permettant ainsi à la narratrice de percer l'opacité et de rêver le monde de l'enfance perdue pour parvenir de façon plus subtile à une naissance poétique. En outre, le silence au milieu de la mer offre à Shamsa la possibilité d'étaler une rhétorique sur ses angoisses, ses émotions et ses origines permettant ainsi de 
réconcilier sous l'égide de l'acte créatif des récits collectifs, des souvenirs sur la terre natale et des récits d'exil. En tout état de cause, le déferlement de l'ombre de la terre natale tout au long de l'espace de La Désirante exprime un détour biographique d'une écriture autorisant l'expression d'une mémoire fragmentaire pour engendrer une poétique de la transcendance dont l'ultime injonction serait l'écartement des barrières culturelles.

Par ailleurs, le recours au mythe d'Ulysse sert toujours de motif pour greffer au texte littéraire un parcours ontologique et caractériser aussi bien l'errance et la déchirure dont sont victimes les personnages d'un roman : erranceentre le lieu ancestral et celui du pays d'accueil que la démarche de connaitre l'Aure. Pour reprendre la pensée de Francesco Sanitra qui discerne en Ulysse la figure typique de l'apprentissage ontologique :

Figure mythologique de l'étranger, Ulysse passe du familier à l'étranger en quête d'un renouveau aussi bien de l'identité que de la reconnaissance [...] Rencontre de l'étranger, de l'inconscient...de deux inconscients. Ecoute de l'autre qui amène le sujet à la découverte de son propre espace, de son propre désir, à travers un voyage comme celui de l'Odyssée. (Anzieu :132)

14 En outre,si donc Ulysse est cet être masculin typique dela mythologie grecque, $L a$ Désirante lui confère désormais une identité féminine. En effet, l'Ulysse masculin est poétiquement converti par le personnageShamsa qui parcourt la Méditerranée pour retrouver Léo disparu depuis huit mois. Une disparition qui ressuscite la mémoire traumatique du personnage principal. Pour mieux saisir le rapprochement entre Ulysse et Shamsa, il est important d'examiner en profondeurles propos de la narratrice :

«Les voiles de Vent de sable ,albatros géant, te cherchent dans le feu du couchant. »(Mokeddem, $2011: 50)$

Ou encore :

» Après cette journée qui inaugure ma navigation en solitaire, je me prépare à la longue veille qui m'attend. » (Mokeddem, $2011: 148$ )

« Ce matin,à l'entrée du détroit de Messine[..]Comme à chaque passage avec toi, j'ai bien sûr repensé aux histoires d'Ulysse. ». (Mokeddem, 2011 : 231-232)

Par ailleurs, les jeux de discours tels qu'ils figurent dans La Désirante permettent d'explorer poétiquement la thématique de l'errance dans l'espace de la mer tout en travaillant un discours concomitant celui inhérent à l'errance dans le désert. Prendre les flots des vagues pour révéler le désert natal édifie la poutre durécit de La Désirante. La mer devient un lieu alternatif qui permet à l'héroïne un rempart vers une reconstruction affective pour fuir les tragédies existentielles. Ainsi, ce lieu permet de créer une alchimie affective chez Shamsa et devient un espace initiatique et métaphore d'exil :

" Je tends mon visage vers les trombes d'eau et de sable et le défie, le fuis. Je pars avec le vent comme à mon premier jour. Mais cette fois,ma fugue et le contraire du bannissement et du renoncement. » (Mokeddem, $2011: 62$ ), proclame Shamsa.

15 De façon générale, la thématique de l'errance, de par la réécriture du mythe d'Ulysse, place le roman de La Désiranteau cœur d'une nouvelle Odyssée allant d'une structure romanesque interne- vu que l'errance est un motif poétique qui refait surface dans la plupart des romans de Malika Mokeddem- vers un voyage dans la mythologie grecque. En outre, le recours au mythe d'Ulysse permet une nouvelle évaluation poétique du concept de l'identité. 


\section{L'errance identitaire et la rencontre culturelle}

16 L'espace de La Désirante est non seulement un motif d'ancrage identitaire mais aussi une passerelle reliant différentes cultures et différents espaces temporels. C'est en fait à ce niveau que l'espace se présente comme la métaphore par excellence de l'errance car il orchestre le passage entre espace de l'enfermement /espace de la liberté, espace réel / espace de la mémoire, espace de la mer /espace du désert, espace d'une quête sentimentale et espace d'une quête identitaire.

Lorsque le récit de La Désirante se charge de dévoiler l'espace tragique où évolue Shamsa une nouvelle vision d'identité s'y fait écho. En fait, la prise de conscience de l'altérité vient du refuge de Misserghine où Shamsaa étérécupérée par des Sœurs blanches.

Plus tard, je me poserai souvent cette question :étais-je une fille des grands espaces ?[...] Mais parce que je ne me sentais appartenir ni à ces sœurs vouées à Dieu ni à celles,ô combien plus nombreuses qui subordonneraient leur existence à la famille[...]'avais l'habitude de courir vers la Méditerranée. Pour faire le vide. (Mokeddem, $2011:$ 51)

17 Face à un naufrage existentiel, l'exil devient un substitut affectif permettant de vivre l'épreuve de soi à soi ainsi qu'une nouvelle maîtrise de la parole. De ce fait, le texte de La Désirante affiche les traces d'un scénario tragique dont l'origine est un abandon menant loin des origines. Tissé sur une trame policière en investissant tout au long du roman l'isotopie de l'enquête, le récit de La Désirante transforme poétiquement une césure identitaire en aventure riche en valeurs humanistes et plonge désormais le roman dans une voie d'apprentissage .En outre, l'espace de la mer se meut en espace où cohabitent les images du passé et constitue un lieu de mémoire révélant métaphoriquement la crise identitairequi a pour cause la séparation précoce de Shamsa d'avec sa famille :

«Il faisait si beau que les bleus de la Méditerranée resplendissent. J'achevais de reconquérir mes repères dans la gloire de leurs rayonnements.» (Mokeddem, $2011: 154)$

Faisant barrages aux pensées extrêmes et aux langages totalitaires, La Désirante de Malika Mokeddem convoque l'espace de la mer pour passer en sourdine le discours sur la figure de la mère et fuir la brutalité du quotidien. En fait, Shamsa fait partager son émotion avec le lecteur en parlant de la mer méditerranéenne :

"La Méditerranée, elle, est comme toutes les mères » (Mokeddem, 2011 : 102-103)

Par ailleurs, la différence culturelle entre Shama et Léo n'est aucunement un obstacle à leur union amoureuse. Bien au contraire, elle est source d'entente et d'apaisement selon Shamsa :

$\mathrm{Tu}$ étais l'Autre, différent et si proche. C'était cette différence qui m'accueillait,m'apaisait et me permettait de m'aimer un peu.[...]Le fait que tu sois absolument étrangerà ce que j'ai vécu jusque là m’a enfin déliée, délivrée de mes inhibitions et de ma déshérence[...]Avant toi j'étais déserte. Notre rencontre m'a rendue désirante. (Mokeddem, $2011: 14$ )

Ainsi, l'expérience de l'amour fait abolir les barrières culturelles et permet de poétiser l'altérité. Dans un autre ordre d'idées, la richesse des références toponymiques dans l'espace du récit de La Désirante place le roman à la confluence de plusieurs cultures car le récit joue sur la notion du voyage aussi bien réel qu'imaginaire.

18 D'autre part, l'espace maritime ainsi que celui du désert renforcent l'isotopie de l'errance caractérisant les œuvres de Malika Mokeddem et offrent aux personnages 
l'opportunité d'exprimer une double identité. Si la quête identitaire se trouve par moments piétinée,Shamsatente de nouvelles expériences et s'engage dans des relations à même de donner une force symbolique à son identité fragilisée.

Ainsi, le métissage culturel permet à la narratrice de La Désirante une expressivité figurative poétisant le questionnement sur soi et sur les autres. En arrivant à libérer sa parole, Shamsa dépasse les contradictions qui l'assiègent et entame sa reconstruction à travers sa relation amoureuse avec un homme étranger. Dès lors, l'épopée de l'amour métamorphose l'épopée des temps anciens pour inventer un espace de métissage culturel. Etant «une fille de grands espaces» amoureuse de la navigation, Shamsa, la journaliste qui tente tant bien que mal de fuir tous les épisodes tristes de son passé, sillonne la Méditerranée pour retrouver son amoureux et reconsidérer ses assises identitaires. Ceci nous amène à dire que le discours sur l'espace propose un traitement poétique et/ou philosophique de l'identité et de la culture.

19 En se fabriquant une fiction celle qui figure la tentative de reconstitution de la scène de la disparition inopinée de son amoureux, Shamsa entretient un récit dédoublé la remontant à ses tragédies anciennes.

Pour sa part, la tension entre la mémoire et l'oubli marque son empreinte dans l'univers fictionnel de La Désirante pouraboutir à une représentation poétique d'un lien parental raté où le retour à l'enfance n'est qu'un préalable pour procéder à une réparation narcissique. Par ailleurs, entretenir un rapport avec un homme étranger permet à Shamsade mieux fixer son histoire personnelle et témoigne d'une demande affective justifiant -constamment -le désir de Shamsa d'aller vers l'Autre et ce, en maintenant en état de constance et de déni son illusion identitaire. Ceci dit, l'éloignement des drames identitaires se conçoit dans l'expérience de l'altérité.

20 D'autre part, l'errance s'entend différemment dans l'espace de La Désirante en prenant tantôt les connotations d'un exil tantôt l'aspect d'un voyage. De ce fait, le voyage entendu, par moments, comme expression d'une errance ciblée et tantôt considéré comme un moment d'égarement permet à la narratrice d'établir un discours sur sa culture et sur celle des autres. C'est dire que l'errance n'est qu'une démarche onirique qui permet une mise en surface d'une expérience traumatique mais aussi une nécessité ontologique pour emboîter le pasà tous les types d'enfermements. En outre, l'évocation du soleil acquiert toute une symbolique dans La Désirante et prend le relais d'affiliation génétique face à un père qui n'a pas assumé son rôle parental :

«La fille de soleil, toi, tu nous reviens.» (Mokeddem, 2011 : 95)

Le père biologique qui est absent dans la vie de Shamsa se substitue alors en un lien imaginaire, de surcroît, un élément du cosmos. Une façon assez révélatrice de la part de l'héroïne de mettre à l'œuvre ses désirs de liberté-en se plaçant dans une généalogie atypique - et un moment de transposer un caractère lumineux sur la vie de ce personnage abandonnique.

\section{Réminisences textuelles dans La désirantre : de l'intertextualité à l'interculturalité}

21 Etudier les œuvres de Malika Mokeddem permet au lecteur d'accéder à un univers romanesque où foisonne la pratique de l'intertextualité tissant dans la trame narrative une symbolique particulière. En effet, la solitude accrue des personnages de Malika 
Mokeddem laisse filtrer un tragique existentiel en connexité avec des expériences plurielles. Dans le creux des souvenirs éparpillés, se dessine désormais au sein de l'espace textuel une intériorité qui se transcende. Pour sa part, le récit polyphonique qui traverse le roman de $L a$ Désirante meuble les artères de la diégèse d'une mémoire à la limite d'une temporalité imprécise et nous revoie non seulement aux œuvres de Camus, aux temps de l'Odyssée homérique mais constitue également un foyer d'échos d'autres œuvres de Malika Mokeddem, à savoir :L'Interdite, N'zid, Mes Hommeset Les Hommes qui marchent. Des œuvres traitantpar excellence la thématique de l'errance.

\section{La Désirante : roman du désert ou Odyssée de la mer?}

22 La Désirante constitue un roman qui accueille de façon signifiante deux isotopies qui paraissent d'emblée divergentes. Il s'agit plus spécifiquement de l'isotopie,rappelons-le, du désert et celle de la mer. Si le désert-lieu d'ancrage identitaire de Shamsa-est un espace castrateur car il participe de l'enfermement moral de l'héroïne, la mer serait cette terre d'exil qui permet à Shamsa de s'affirmer en tant que sujet et d'entamer ses multiples rebellions :

" Ain Dakhla signifie " la source de l'entrée " Heureux ce premier jour où j'ai quitté

cette terre exilée en elle-même » (Mokeddem, $2011: 26$ ), affirme Shamsa.

En outre, les voyages de Shamsa à travers le bassin méditerranéen consolident la dimension intertextuelle de La Désirante.En effet, le roman retrace l'aventure de Shamsa dans le large méditerranéen et regorge de références à un décor qui nous renvoie au monde de l'Odyssée telles queles références à Ithaque, Céphalonie, Camargue, Charybde, Scylla, l'île de Calypso.

\section{Du nom de Shamsa à son rapport à la mer : le soleil et la mer de Camus?}

23 L'article en question ne prétend guère procéder à une étude comparative entre $L a$ Désirante et l'œuvre poétique d'Albert Camus. Cependant, le dévoilement de certains thèmes dans notre roman, à savoir le soleil, l'absurde, la mer et la relation douloureuse avec la mère nous renvoientindubitablement à certains symboles récurrents dans les œuvres de cet écrivain français (31). En effet, l'univers de La Désirantemet en exergue une interconnexion des symboles tels le soleil et la mer. Deux symboles qui prédestinent Shamsa dont le nom signifie Shams ou soleil en langue arabe à être »Une fille des grands espaces. "

C'est ainsi que la mer devient comme dans l'univers d'Albert Camusle symbole del'affranchissement et dela sérénité :

» Nulle part ailleurs, je n'avais ressenti cette plénitude. L'impression d'avoir enfin trouvé ma place dans ce berceau flottant entre deux rives. Une coque de plastique pour des amours bercés par les bleus de la Méditerranée. » (Mokeddem, 2011 : 32), confie Shamsa dans son journal intime.

24 En voulant donner de la lumière à sa vie morne, la narratrice de La Désirante s'approprie un élément du cosmos qu'est le soleil. Par ailleurs, la quête de la figure maternelle se fait entendre dans le roman de Malika Mokeddem par le recours au symbole de la mer un autre motif littéraire cher à Camus. 


\section{Les jeux de discours entre contextualisation et errance}

25 L'analyse des paroles des personnages du roman de La Désirante révèle une dialectique linguistique porteuse d'une hybridité discursive. Ainsi, les mots et les phrases sont fréquemment intégrés dans une démarche traductrice naturellement assumée par la narration qui est révélatrice de la double identité culturelle des personnages du roman de Malika Mokeddem.En rapport avec le récit de la mémoire, le discours des personnages de La Désirante est porteur d'une diversité langagière et culturelle. Ainsi, l'héroïne- narratrice de La Désirante existe à travers sesvoyages mais aussi à travers ses témoignages sur ses différentes histoires.

En outre, le discours des personnages emprunte plusieurs directions lorsqu'il s'agit de poétiser la contingence ou d'étaler une rêverie dans les temps anciens ou dans les imaginaires lointains. De même, le discours de Shamsa devient compréhensif lorsqu'il passe du présent au passé et du passé au présent puisqu'il s'agit foncièrement d'un récit de mémoire.

26 D'autre part, l'écriture de Malika Mokeddem s'inscrit dans le sillage d'une origine culturelle bédouine et de la culture du pays d'accueil.Cette hybridité se traduit sur le plan linguistique par l'interférence des langues. En effet, le lexique arabe fait corps avec la langue française dans le roman La Désirante pour permettre un métissage linguistique qui dévoilerait tantôt un particularisme révélateur tantôt une dimension interculturelle.

Plus précisément, le brassage linguistique s'effectue aussi bien au niveau onomastique car le roman confectionne des noms dont l'origine est arabe ou berbère tels que :» Zin", "Zineb", "Nabil », "Youcef», "Kader », " Aicha ». Aussi, pourrions nous déceler des références à des spécialités culinaires locales "Méchouia ", "Hrira ", "Couscous », etc. Nous avons également à noter des références à la toponymie telles « Ain Dakhla », "Tanezrouft », » Misserghine », etc. En outre, La Désirante de Malika Mokeddem est à lire comment un récit où l'on perçoit des bribes d'un discours social car on y relève des références à la culture religieuse de la narratrice à travers des expressions à l'exemple de :» inch'Allah », «Coran », « Muezzin », « kaffer »,etc .Le texte de La Désirante insère également certains clichés et expressions de la culture algérienne,à savoir « jiâne »,» ouleds ", " zarga », » baraka »,» chouia », » chiche ", » chi-chi ». ${ }^{6}$

27 Il en découle que le bouillonnement des langues dans le texte de La Désirante est loin d'être exclusivement un choix linguistique anodin mais il reste typiquement relié au plurilinguisme de l'auteure du roman ainsi qu'à une orientation scripturairequi projette l'œuvre littéraire dans une dimension polyphonique pour faire du roman la réverbération de plusieurs jalonnements aussi bien linguistiques que culturels. La Désirante de Malika Mokeddem est ainsi un texte générateur d'une organisation narrative dont l'imaginaire du personnage principal inhibe la linéarité et accrédite la fragmentation pour faire passer la nécessité de l'errance et l'expression de l'identité en bribes. 


\section{La désirante : de la prose textuelle à l'esthétique des mélanges génériques}

28 En plus de son style simple, l'œuvre de Malika Mokeddem dépasse sa dimension autobiographique où la narration est prise en charge par un "Je" constant pour basculer l'énonciation à elle "Shamsa " et ouvrir la voie à des pratiques textuelles qui font de La Désirante un roman dialogique car plusieurs textes viennent à s'y greffer.

Par ailleurs, le programme fictionnel de La Désirante emprunte un style d'écriture évinçant le principe du cloisonnement générique. Bien que l'inscription "roman» figure clairement dès la page de couverture, la lecture de toute l'œuvre nous fait constater d'autres vérités. En effet, à l'image de l'identité hybride du personnage principal, La Désirante obéit à une stratégie scripturaire particulière et offreun mélange générique signifiant. Ainsi, $L a$ Désirante-étant foncièrement un roman de la mémoire- se donne à lire comme un récit pulvérisant différentes strates du discours. De ce fait, le nomadisme intérieur dont se réclame Shamsa fait de la narration un espace essentiellement fragmentaire où différentes écritures viennent à s'y agripper. Cohabitation de l'épique et du dramatique, $L a$ Désirante demeure un roman portant le soliloque d'une voix narrative qui, par la mise en scène d'un réinvestissement franchement intense d'un conflit intersubjectif, mis à nu de par l'expérience d'introspection, interroge ses zones d'ombre et aspire à interposer une parole qui donnerait, in fine, une réplique à sa plénitude.Le partage énonciatif, dans l'espace du récit de La Désirante, entre Je et Tu,donne à flotter, semble-t-il, un sentier discursif laissant spontanément déferler l'épreuve de la dialectique de l'altérité.

29 Entre monologue intérieur retraçant les différents soliloques de Shamsa, roman policier car il narre les aventures de cette héroïne à travers la Méditerranée pour élucider les circonstances de la disparition de son compagnon Léo, journal du bord vu que la narratrice annote l'itinéraire de ses passages en Méditerranée, journal intime retraçant le passé de Shamsa, roman de témoignage qui s'arrête sur une conjoncture historique relative à la décennie noire dans les années 90 en Algérie, roman de voyage où le voyage convoite en parallèle l'exil, roman-conte relatant sous forme d'un conte féérique l'abandon de Shamsa dans le désert algérien, fresque d'amour ou roman sentimental puisqu'il s'agit aussi d'une quête des amours perdues etautofiction de par les similitudes avec la vie de l'auteure, le roman de Malika Mokeddem vient briser la question de l'unité générique et se donne à lire comme une œuvre qui se caractérise par l'enjambement des modèles génériques à même de consolider la notion de l'errance textuelle tant travaillée dans La Désirante.

Tout le roman s'accorde en fait avec cette philosophie de l'errance. Entre récit intime composant avec une contingence historique locale vu que l'auteure aborde en filigrane l'Histoire de son pays et récit ouvert abritant plusieurs quêtes, La Désirante reste un roman d'exil et d'errance tentant d'exprimer par le biais de la métaphore cette peur de se cloitrer dans des filiations ratées. En errant de façon incessante, Shamsa tente de surpasser ses néants identitaires pour s'affirmer dans l'expérience de l'amour.

Par ailleurs, de la mémoire perdue à la mémoire recouverte, le dévoilement de soi à travers l'écriture comporte un impérieux désir de parcourir les lieux et les imaginaires. Ainsi, l'expression de l'hybridité enrobe tous les éléments discursifs du récit de $L a$ Désirante et pénètre l'architecture textuelle. Somme toute, en faisant dans la fracture 
générique, Malika Mokeddem propose une esthétique qui arracherait son roman à la tyrannie de la forme et pose d'emblée les jalons identitaires de son écriture.

N'ayant jamais eu de repères de sa vie d'enfance, l'errance dans soi et vers les autres permet à la narratrice du roman de La Désirante une transmutation existentielle suite à sa quête continuelle pour trouver des réponses à des interrogations plurielles qui rythment son existence. D'une part, il y'aurait dans La Désirante un désir irrésistible d'aller au bout d'une quête du sujet. D'autre part, on y discernerait un impérieux besoin de la relancer. Quantà la réécriture du mythe d'Ulysse et à l'inscription de la thématique de l'errance dans le roman, nous avons pu noter un rapport philosophique à l'intertexte mythologique puisqu'il s'agit pour l'auteure du roman ainsi que pour la narratrice d'établir non seulement une connivence artistique avec un mythe tant représenté dans les écritures littéraires mais aussi d'incarner par l'entremise des pratiques inter et /ou intratextuelles une réflexionontologique à travers une poétique abordant savamment la pensée universelle et la théorie de l'altérité. En cherchant de rassembler les fragments de sa mémoire désintégrée, Shamsa agence à son récit hybride un humanisme nouveau et ce, à travers son errance dans des espaces réels ou imaginaires.

Ceci posé, la narratrice de LaDésirantelaisse entendre à travers un dispositif énonciatif percutant une double quête dont l'une consisterait à trouver un sens existentiel et dont l'autre concrétiserait le sens textuel par le biais d'une écriture de l'intime passant de l'oralité à l'écriture.

31 Ainsi, la profondeur de la quête de Shamsa impose la nécessité de la transcendance. D'autre part, profusément errante, la narratrice, sous le couvert du mythe d'Ulysse, étend en filigrane une nouvelle poétique qui arrache progressivement le monologue intérieur au récit de la mémoire. Dans cette œuvre où s'exprime une tension fondamentale entre l'errance et la mémoire, se dessineun désir d'atteindre la cohérence ainsi qu'une éthique de la sagesse déracinant progressivement l'héroïne de son vide identitaire pour dresser la métaphore de l'altérité et /ou un monde humaniste sans quoi l'Homme ne serait qu'étranger à lui-même. Ce faisant, La Désirante,enconférant des accents mythiques au vent du désert,reste l'odyssée scripturaire initiatique qui s'enquiert d'une alchimie spatiale et d'une errance entre les cultures.En évoquant le poème de Saint-John Perse, l'auteure de ce roman tente d'alterner les déserts identitaires aux épreuves d'altérité pour mieux transcender les frontières et se réconcilier avec l'espace perdu. Autrement dit, c'est grâce à la destruction des frontières spatiales et à l'ouverture aux imaginairesque la narratriceShamsa-dont le nom la destine à intégrer la lumière-peut communiquer ses manques et ses exiles et légitimer ses appréhensions identitaires en les intégrant subtilement dans son journal intime. N'étaient-ce pas l'écriture journalistique et d'autres écritures auxquelles s'adonnait Shamsa qui lui permettaient d'harmoniser» un réel » des plus effroyables? Autrement dit, c'est par le biais de l'écriture que les épreuves traumatiques livrées par la narratriceShamsa -à l'aune du mythe-sont inscrites dans une nouvelle démarche vers le bonheur. Ceci nous amène à affirmer qu'à travers le roman La Désirante, Malika Mokeddem présente à souhait l'errance, la perte et le métissage comme matières philosophiques $\mathrm{du}$ texte généraloù le vrai évènement du récit-cadre est le voyageontologique juxtaposé à l'expérience de la résiliencepour suggérer une conversion de la quête de la narratrice Shamsa. Cela reste également une manière habile chez 
l'écrivaine pour inscrire le mythe d'Ulysse dans un nouveau mouvement d'écritureouvrant la voie au rapprochement de plusieurs imaginaires.

\section{BIBLIOGRAPHIE}

Loin d'être une simple reprise de la thématique de l'errance, on peut dire que le recours au mythe d'Ulysse dans La Désirante réconforte plutôt la thèse du non retour et non celle du retour. En effet, étant inscrite dans le nomadisme, Shamsa se recueille dans un nouvel espace-temps, construit une identité sans clôture où le concept derridien de la destruction prend toute sa considérationet largue à nouveau les amarres pour braver les frontières.

Amrani Mehana, La poétique de Kateb Yacine. L'autobiographie au service de l'Histoire,L'Armattan,2012.

Anzieu Didier et al.Différencc culturelle et souffrance de l'identité, Dunod,Paris,1998,p.132.

Blanchot Maurice, L'espace littéraire, Folio/Essai, Gallimard, 1995.

Bonn Charles, Echange et mutations des modèles littéraires entre Europe et Algérie, L'Harmattan, Paris, 2004.

Bonnefoy Yves, Dictionnaire des mythologies, Paris, 1981.

Bouchet René, Le nostalgique : L'imaginaire de l'espace dans l'œuvre d'Alexandre Papadiamantis, Presses de l'Université de Paris-Sorbonne, Paris, 2001.

Brunel Pierre (dir.), Dictionnaire des mythes d'aujourd'hui, éd. Du Rocher, 1999.

Brunel Pierre, Mythocritique, théories et parcours, Paris, Puf, 1992.

Brunel, Pierre, Dictionnaire des mythes littéraires, Nouvelle édition argumentée, Editions Du Rocher, 1988.

Canvat Karl, Enseigner la littérature par les genres .Pour une approche théorique et didactique de la notion de genre littéraire, De Boeck \&Duclot, 1999.

Derrida in Les grands philosophes,Ellipses,2014.

Durand Gilbert, Figures mythiques et visages de l'œuvre : De la Mythocritique à la mythanalyse, Dunod, Paris, 1992.

Ferréol Gilles\&Jucquois Guy, Dictionnaire de l'altérité et des relations interculturelles,Armand Colin,Paris,2003,p.175.

Freud Sigmund, L'inquiétante étrangeté et autres essais, Folio essais ,Gallimard,1998.

GafaïtiHafid, Les femmes dans le roman algérien, L'Harmattan, Paris, 1996.

Gasparani Philippe, Est-il je ? Roman autobiographique et autofiction, Seuil, Paris, 2004

Gassin Jean,L'univers symbolique d'Albert Camus, essai d'interprétation psychanalytique, Librairie Minard,1981, p. 33.

Genette Gérard, Seuils, Paris, 1987. 
Hubier Sébastien, Littératures intimes. Les expressions du moi, de l'autobiographie à

l'autofiction, Armand Colin, Paris, 2003.

Huet-Brichard Marie-Catherine, Littérature et mythe, éd, Hachette Supérieur, 2001,p.04.

kristeva Julia, Etrangers à nous-mêmes, Folio essais,Gallimard,1991.

Léonard-RoquesVéronique,Figuresmythiques.Fbrique et métamorphoses,Presses Universitaires Blaise Pascal, Clermont-Ferrand,2008.

Mokeddem, Malika, La Désirante, Casbah Editions, Alger, 2011.

Nasta Dan-ion, Saint-John Perse et la découverte de l'être,Presses Universitaires de France,Paris, 1980

Paravy Florence, L'espace dans le roman africain francophone contemporain (1970-1990),

L'Harmattan, Paris, 1999.

Philibert Myriam, Dictionnaire des mythologies, Maxi-Poche,2002

Piegay-Gros, Nathalie, Introduction à l'intertextualité, Dunod, Paris, 1996.

Thaâlbi, Ben Meziane, L'identité au Maghreb, l'errance, Alger, Casbah Editions, 2000.

Visonneau Geneviève, L'identité culturelle, Armand Colin, Paris, 2002.

\section{NOTES}

1. Voir Piegay-Gros, Nathalie, Introduction à l'intertextualité, Dunod, Paris, 1996 : pp. 7-8.

2. Je m'appuie principalement sur la notion de l'étarngeté empruntée à Freud chez Kristeva Julia dans Etrangers à nous-mêmes, Folio essais,Gallimard,1991.

3. Voir le motif du vent chez Nasta Dan-ion, Saint-John Perse et la découverte de l'être,Presses Universitaires de France,Paris,1980.

4. Voir Genette,Gérard,Seuils,Essais,2002.

5. Voir Ferréol Gilles\&Jucquois Guy, Dictionnaire de l'altérité et des relations interculturelles, Armand Colin,Paris,2003 :p.175.

6. Malika Mokeddem emploie ces expressions empruntées à l'arabe dialectal dansLa Désirante, Casbah Editions, Alger, 2011.

\section{RÉSUMÉS}

Dans cet article,nous nous intéressons au rapport du discours ontologique à l'expression du mythe d'Ulysse dans La Désirante (2011) de Malika Mokeddem. En effet, dans ce roman, toutes les occurrences mythologiques sont étroitement liées à la quête de l'héroïne-narratrice, Shamsa, qui se révèle être une quête de soi. Dans ce sens, notre travail ne consiste pas à dresser, stricto sensu, un inventaire des traits figuratifs du mythe d'Ulysse, ce qui serait à notre sens sans intérêt critique, il est plutôt question de définir les enjeux signifiants du recours de l'écrivaine au dit mythe pour concevoir la vision du monde de l'héroïne-narratrice et tracer son parcours romanesque à travers lequel se dessine son chemin de la réappropriation de sa mémoire. Dores et 
déjà, nous situons la problématique du présent articledans l'examen des modalités de la reprise de l'intertexte mythologique et des messages qui en sont accolés et ce, en fondant notre analyse sur les concepts d'intertextualité et de l'errance.

In this article, we are interested in the report of the ontological discourse on the expression of the myth of Ulysses in Malika Mokeddem's La Désirante (2011). Indeed, in this novel, all the mythological occurrences are closely linked to the quest for the heroine-narrator, Shamsa, who turns out to be a quest for oneself. In this sense, our work does not consist in training, strictly speaking, an inventory of the figurative features of the myth of Ulysses, which in our opinion would be without critical interest, it is rather a question of defining the significant issues of the writer's recourse to the said myth to conceive the vision of the world of heroin- narrator and trace his romantic journey through which draws his path to the reappropriation of his memory. We are already situating the problem of the present article in the examination of the modalities of the resumption of the mythological intertext and the messages which are attached to it, and this, basing our analysis on the concepts of intertextuality and wandering.

\section{INDEX}

Mots-clés : intertextualité, mythe, réécriture, Ulysse, altérité

Keywords : intertextuality, myth, rewriting, Ulysses, otherness

\section{AUTEURS}

\section{SABRINA TOUNES YAMOUNI}

Université Abderrahmane Mira, Bejaia Algérie

\section{SOUHILA OURTIRANE RAMDANE}

Université Abderrahmane Mira, Bejaia Algérie 